\title{
Analysis of Smart Applications using Internet of Things
}

\author{
B R Vatsala ${ }^{1}$, Dr C Vidyaraj ${ }^{2}$ \\ Assistant Professor, Department of CS\&E, The National Institute of Engineering, Mysore, India ${ }^{1}$ \\ Professor, Department of CS\&E, The National Institute of Engineering, Mysore, India ${ }^{2}$
}

\begin{abstract}
Internet of Things which is a network of physical objects plays a vital role in the near future. The idea behind Internet of Things is that Things instead of humans can make use of Internet to communicate. IoT can be used to ease the life style of people with better facilities such as smart cities, homes, hospitals that give a better service than the traditional one. Analysis of applications that use IoT is very much needed to improve the quality of life style of people.
\end{abstract}

Keywords: Internet of Things, Motes, WSN Nodes, Smart Cities, Smart Homes, Smart Hospitals, Ontology

\section{INTRODUCTION}

The IoT consists of billions of digital devices, people, services and other physical objects having the potential to seamlessly connect, interact and exchange information about themselves and their environment [1]. It will combine the power of universal network connectivity with embedded systems, sensors and actuators in the physical world. It allows devices to communicate with each other, access information over the Internet, store and retrieve data and interact with users in smart, pervasive and always connected environments. Internet of Things plays a vital role in the near future. People want their work to be done quickly with ease. The idea behind Internet of Things is that Things instead of humans can make use of Internet to communicate making life easy. The concept of Smart Homes, Smart Cities, Smart Hospitals are all based on Internet of Things. This paper discusses the usage scenarios along with design strategies.

This paper is organized as follows. Section II focuses on usage of Internet of Things. Section III discusses the applications of IoT in Smart Cities Section IV discusses the applications of IoT in Smart Homes. Section V discusses the applications of IoT in Smart Hospitals.

\section{USAGE OF IOT}

The scenarios where IoT can be used are in developing Smart Cities, Smart Homes, Smart Hospitals, Smart Offices, Smart Transportation, Military Systems, Smart banks, Logistics Applications, Business and many more.

A. Applications of IoT at Smart Cities:

Some of the application scenarios of IoT in smart cities include

- Automatic Parking Management.

- Stationary Structure Monitoring

- Traffic Monitoring

- Environment Monitoring

B. Applications of IoT at Smart Homes:

Some of the application scenarios of IoT in smart Homes include

- Locking of all windows and doors from a central location in the home.

- Opening and Closing of doors when member of the home enters and leaves home.

- Turning on and off of Lights when someone enters and leaves a room, in response to the amount of daylight entering the home.

- Setting refrigerators, heaters, air-conditioners, ventilators etc to automatically change in response to the time of day or to external temperatures

- Alerting home owner to unusual activity, through sensors that evaluate foot-pressure changes, breaking glass, movement, contact, Gas leakage, fire etc

- Detecting high temperatures and automatically shutting off burners and ovens.

- Controlling Audio to allow music to be heard in one or more rooms of the house, Interconnecting televisions and media sources 
- Room-to-room video and audio communication systems allow viewing and talking among people in different rooms and floors of the home.

C. Applications of IoT at smart hospitals

Some of the application scenarios of IoT in smart hospitals include

- Monitoring Health condition such as Blood pressure, Diabetic status, Cardiac Activity etc of the patient at remote place.

- Automatic detection of Wound healing and applications of medicines based on status of wound

D. Applications of IoT at Smart Transportation Systems

Some of the application scenarios of IoT in smart transportation include

- Automatic Driving

- Setting Accident free Transportation Systems

- Automatic Traffic Monitoring Systems

E. Applications of IoT at Military

Some of the application scenarios of IoT at military include

- Automatic surveillance systems

- Automatic launching of missiles

- Detection of mass destructors

F. Applications of IoT at Banks

Some of the application scenarios of IoT at banks include

- Cashless transactions

- Balance enquiry systems

\section{APPLICATIONS OF IOT AT SMART CITIES}

Smart Cities are of major concern especially in developing Countries like India where the resources are to be managed potentially because of increased population leading to pollution, traffic, parking problems etc the smart city project in India costs Rs 131,762 Crores [10] IoT can be used for developing smart cities. Design strategies of Parking management, Structure monitoring, traffic monitoring and environment monitoring are discussed below

\section{A. Automatic Parking Management:}

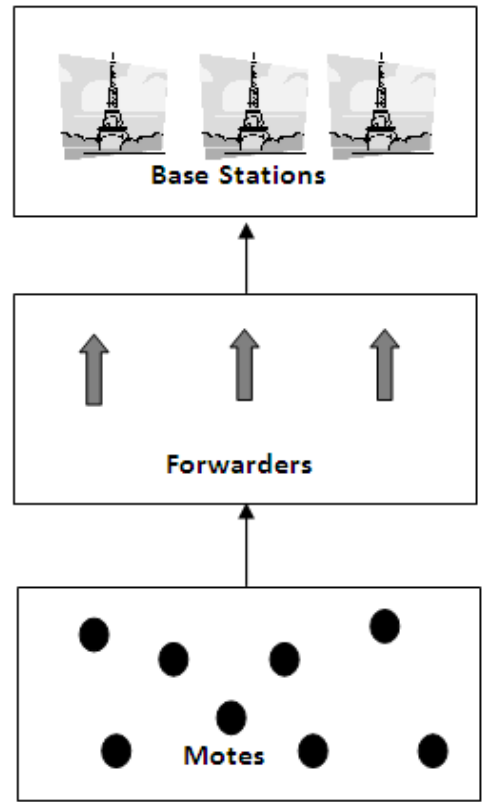

Fig. 1 Automatic Parking Management System Design

Automatic parking management [2] is the best service the corporation can provide to the citizens. The parking bay occupancy details can be collected by sensors and sent to the smart phones of the users. It will also enable the 
Corporation to induce fine in case of parking infringements. some commercial systems are available based on this wireless technology [9]. These systems work autonomously in three tier mode where the lowest tier motes are attached to sensors which are glued to the ground, the middle tier contains forwarders connected to light poles and the uppermost tier contains base stations connected to an Internet enabled device [3]. Fig 1 demonstrates this system.

B. Stationary Structure Monitoring: A city may consist of stationary structures - such as huge buildings, dams, Towers, Fly overs or bridges [4]. The status of these large structures is very critical any damage to these structures may cause life-threatening situations and serious financial loss. WSN nodes can be embedded in these structures which send information to sink nodes about their status. The data collected at the sink are then analysed to detect abnormality and any damage that may cause due to this may be prevented, Fig 2 demonstrates this system.

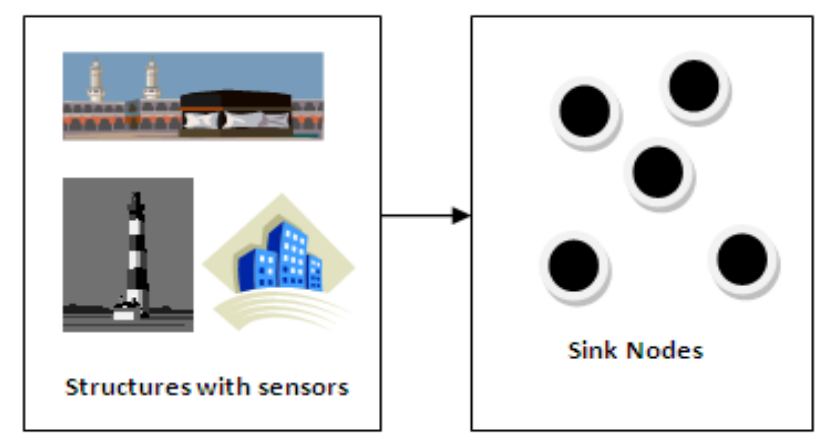

Fig. 2 Structure Monitoring System Design

C. Traffic Congestion and Impact Monitoring: Traffic Congestion is the major contributor to noise pollution and air quality pollution Traffic congestion directly imposes significant costs on economic and social activity in cities. congestion in India is increasing day by day This causes significant delay in time management and degrades the performance of citizens as most of the time is wasted in traffic especially in metropolitan cities. There are a variety of sensors available for measuring pollution levels and traffic delays and queuing using vehicle-to-vehicle (V2V) and vehicle-to-infrastructure (V2I) [8] communications, sensors form ad-hoc vehicular networks, which allow online monitoring of travel times, origin-destination route choice behavior, queue lengths and air pollutant and noise emissions, as well as predict possible accidents. This valid information on traffic conditions can be presented to travellers (smart phones) which help travellers to choose shortest temporal route.

D. Compressive Sensing for Environmental Monitoring: Environment monitoring is very important as it impacts on health of people. Large scale environmental monitors can be developed to detect parameters such as hydrocarbons, oxides of nitrogen photochemical smog, carbon-dioxide, carbon-monoxide, ammonia and benzene and take preventive action in case of any abnormalities. Microclimate sensing can also be achieved through the deployment of temperature and humidity sensors to improve the quality of life and productivity for a more sustainable city.

\section{APPLICATIONS OF IOT AT SMART HOMES}

The aim of smart home systems is to create an environment that is aware of the activities taking place within it. Monitoring of the home is very essential for example most of the day time the all family members will be away from home due to engagements in work, studies etc a small leakage of gas may result in dangerous hazard, running taps, lights that are on lead to wastage of precious resources. Smart Home system monitors the electrical energy consumption based upon the real time tracking of the devices at home. The electrical devices and switches can be remotely controlled and monitored [5]. Which helps in good utilization of electrical power. It uses various sensors to not only monitor the real time device tracking but also maintaining the security of the house. Smart Home also concentrates on disabled people to make their life easier as they encounter with a lot of difficulties in their everyday life even when they are at home.

The Design of Gas Leakage System and Door Control Systems cases are discussed below.

A. Gas Leakage System Design: Gas leakage leads to dangerous hazards to detect it gas sensors such as MQ-6 which detects dangerous flammable gas can be used. It has high sensitivity to propane, butane and LPG, also response to natural gas. It is has low cost and suitable for different application. Gas detection circuit (Source Node) is prepared with MQ-6 gas sensor. It gives a simple on/off signal. When it detects a gas leaking it gives logic high to its output pin. 
The data at the output pin is transmitted to Intermediate node, Upon getting High Signal It transmits signal to Gas Controller (Sink Node) to shut off the gas regulator.

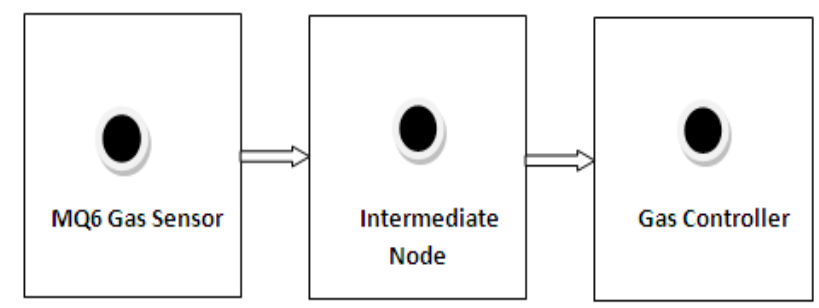

Fig. 3 Gas Detector System Design

B. Door Control System Design: Door Control systems are mainly designed for disabled people who face a lot of problem for locking and opening the door. Door Control Node (Sink node) and two Motion Sensors (Source nodes) are placed at the door frame. There is a computer (intermediate node) in which home automation program is running. Each member of the house has an id which is recorded in the database. The member of the house should carry a preprogrammed WSN Node. When Member of the house comes near the door the by the presence of WSN node he wears Motion Sensors node detects and sends the id to the Computer, the home automation program running in the computer sends signal to Door control Node to open or close the door if the id is valid.

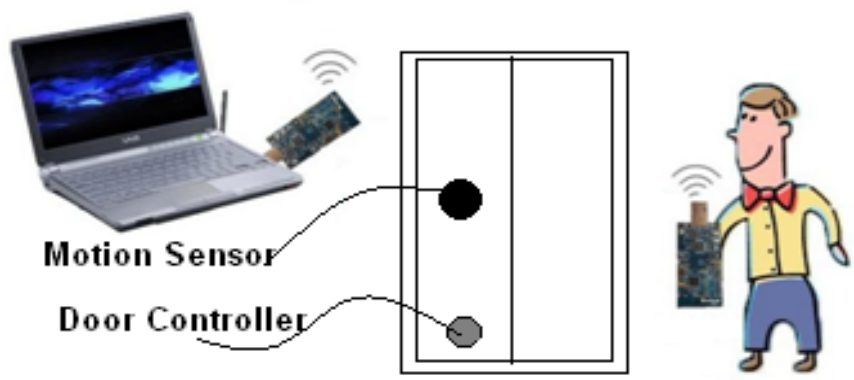

Fig. 4 Door Control System Design

\section{APPLICATIONS OF IOT AT SMART HOSPITALS}

Smart Hospitals are built by focussing on patient's experience, employee and organization. Smart hospitals play a vital role in rehabilitation of patients and Helps patients to get timely service needed It also helps doctors to treat his patients even when they are away from patients.

Rehabilitation and wound healing process detection cases using IoT are discussed below

A. Rehabilitation using IoT: Rehabilitation helps an individual to gain greater independence after illness, injury or surgery to accomplish rehabilitation patients' symptoms are analyzed, diseases are located and all the information are put into a remote database through internet [7]. When the patient first enters the hospital, the characteristics of the disease are determined by the doctor. In this process, several factors such as patients' basic information and disease condition are stored in the remote database in form of ontology through networks. also The personalized preferences such as least cost, best treatment are found and stored.

After that the patients' conditions are compared with the stored cases in the remote data base with corresponding successful rehabilitation strategies in the disease ontology. Based on the successful strategies, more detailed rehabilitation strategies are worked out, such as type of walking training, Duration of treatment activity etc. in addition to medical activity, The information regarding each medical resource in the medical resources ontology is also determined. Resource allocation plans are worked out depending on the information provided by the IoT. After this each patient is well diagnosed and best rehabilitation strategies are possessed which include the basic treatment activity information and resources allocation plan.

B. Wound Healing Process Detection: Wound Healing process is very important dynamic process. The status of the wound is to be monitored periodically to decide the medication policy. Traditionally wound dressing is removed 
for inspection which may lead to infection and skin irritation. Instead of traditional method Bio-Sensors are used to detect the wound healing stage. Proteases such as neutrophil elastase which is wound healing enzyme is detected using a lab-designed FRET-based protein biosensor fig 5 demonstrates this system. Thus without removing wound dressing status of the wound can be analysed [11].

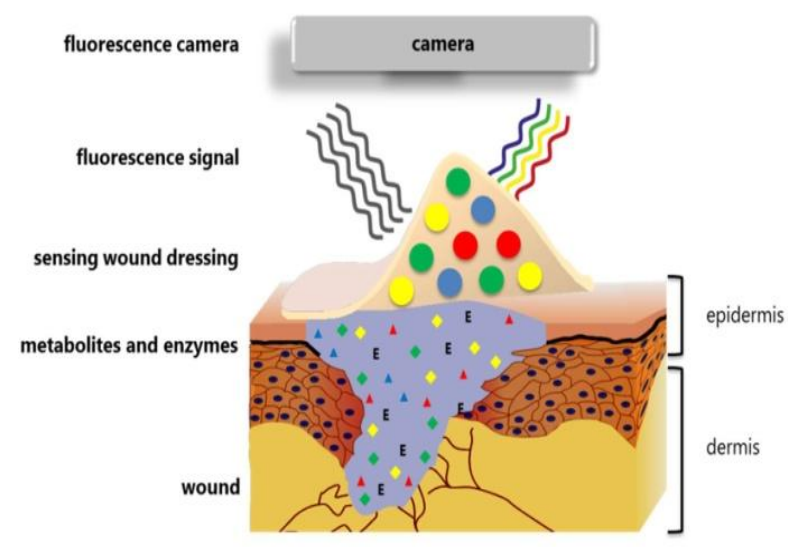

Fig. 5 Wound Healing Process detection System Design

\section{CONCLUSION}

Various Applications where IoT can be used and its future is analyzed along with case studies of Parking management, structure Monitoring, traffic monitoring, environment Monitoring at Smart Cities, Gas leakage systems and door control systems at smart homes and rehabilitation and wound healing process detection at Smart Hospitals. Thus IoT is the future of Internet providing quality of life.

\section{ACKNOWLEDGMENT}

We acknowledge the support of the National Institute of Engineering in the completion of this work and also acknowledge authors whose paper has been referenced.

\section{REFERENCES}

[1]. Charith Perera, Arkady Zaslavsky, Chi Harold Liu, Michael Compton, Peter Christen, and Dimitrios eorgakopoulos, "Sensor Search Techniques for Sensing as a Service Architecture for the Internet of Things", IEEE SENSORS JOURNAL, VOL. 14 , Feb 2014.

[2]. Jiong jing,Jayardhana Gubbi,Slaven Marusic, Marimuthu Palaniswamy, “An Information Framework for Creating a Smart City through Internet of Things"'IEEE Journal PP 2327-4662,2013

[3]. S. Lee, D. Yoon, and A. Ghosh, "Intelligent parking lot application using wireless sensor networks," in Proceedings of International Symposium on Collaborative Technologies and Systems (CTS), 2008, pp. 48-57.

[4]. J.-P. Vasseur and A. Dunkels, Interconnecting Smart Objects with IP: The Next Internet. Elsevier, 2010.

[5]. Himanshu Varma, Adithya Vikram, Madhu Jain, Gaurav Varma," Smart Home System Based on Internet Of Things", IEEE ,2016

[6]. Jasmeet Chhabra and Punit Gupta, "IoT based Smart Home Design using Power and Security Management", 1st International Conference on Innovation and Challenges in Cyber Security (ICICCS), 2016.

[7]. Yuan J. Fan, Yue H. , Li D. XuYan Zeng, Fan Wu "IoT Based Smart Rehabilitation System" IEEE transactions on industrial informatics, vol. 10, no. 2, may 2014

[8]. Wu He, Gongjun Yan and Li Da Xu "Developing Vehicular Data Cloud Services in the IoT Environment",IEEE transactions on industrial informatics, vol. 10, no. 2, may 2014

[9]. http://smartgrains.com.

[10]. http://smartcities.gov.in

[11]. Dagmara Jankowska, Cindy Schulenburg, Greta facio, Katharina Maniura, Michael Richter ,"Biosensors for monitoring of enzymatic wound healing processes” 10th World Biomaterials Congress, Montréal, Canada, 17 May - 22 May, 2016. 